\title{
Niclosamide inhibits colon cancer progression through downregulation of the Notch pathway and upregulation of the tumor suppressor miR-200 family
}

\author{
MOHAMMED A. SULIMAN ${ }^{1 *}$, ZHENXING ZHANG $^{1,4^{*}}$, HEYA NA $^{1}$, AILTON L.L. RIBEIRO ${ }^{1}$,

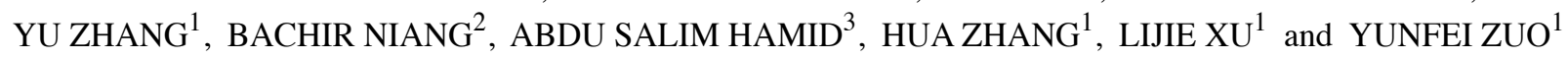 \\ ${ }^{1}$ Department of Clinical Biochemistry, Dalian Medical University, Dalian, Liaoning 116044; \\ ${ }^{2}$ Department of Biochemistry, Institute of Glycobiology, Dalian Medical University, Dalian, Liaoning 116044, \\ P.R. China; ${ }^{3}$ Department of Clinical Laboratory Science, Asmara College of Health Sciences, Asmara, \\ Eritrea; ${ }^{4}$ The First Clinical College, Dalian Medical University, Dalian, Liaoning 116044, P.R. China
}

Received November 29, 2015; Accepted June 23, 2016

DOI: $10.3892 / \mathrm{ijmm} .2016 .2689$

\begin{abstract}
Colorectal cancer (CRC) is among the most frequent causes of cancer-related deaths worldwide. Thus, there is a need for the development of new therapeutic approaches for the treatment of CRC. Accumulating evidence has revealed that niclosamide, an anthelminthic drug, exerts antitumor activity in several types of cancer, including colon cancer. However, the underlying molecular mechanisms responsible for the effects of this drug remain elusive. Previous studies have shown that the aberrant Notch signaling pathway contributes to the carcinogenesis of colon cancer. Herein, we examined the effects of niclosamide on the growth, migration and apoptosis of colon cancer cells, and the role of the Notch signaling pathway. By performing MTT, wound-healing and Transwell migration assays, we observed that niclosamide suppressed the growth and migration of colon cancer cells, and flow cytometry demonstrated that cell apoptosis was induced. This was associated with the decreased protein expression of Notch1, Notch2, Notch3 and Hey1, and the increased expression of the tumor suppressor microRNA (miR or miRNA)-200 family members (miR-200a, miR-200b, miR-200c, miR-141 and miR-429) that are typically downregulated in colon cancer. Collectively, these findings demonstrate that niclosamide potentially inhibits the progression of colon cancer by downregulating Notch signaling and by upregulating the miR-200 family members.
\end{abstract}

Correspondence to: Professor Yunfei Zuo, Department of Clinical Biochemistry, Dalian Medical University, Dalian, Liaoning 116044, P.R. China

E-mail: zyf04112002@dlmedu.edu.cn

${ }^{*}$ Contributed equally

Key words: Notch signaling, niclosamide, apoptosis, colon cancer, miRNAs

\section{Introduction}

Colorectal cancer (CRC) has become a major clinical problem and ranks as the third most prevalent cancer and the fourth leading cause of cancer-associated death worldwide (1). This is due to the limitations of chemotherapy as a result of drug resistance and organ toxicities (2). To overcome these serious limitations, the design of novel approaches targeting cancer-related signaling pathways is key to improving treatment outcomes in colon cancer.

The Notch signaling pathway plays a vital role in several cellular processes. Notch signaling is considered to play an oncogenic role in the pathogenesis of CRC $(3,4)$. Previous studies have also revealed that Notch signaling is deregulated in several types of human tumors, including colon cancer $(5,6)$, which implies that screening various chemotherapeutic agents for their anti-colon cancer potency by targeting the Notch pathway may be extremely valuable. In humans, the Notch signaling pathway has four receptors (Notch-1, -2, -3, and -4) and five ligands (Jagged-1, Jagged-2, Delta-1, Delta-3 and Delta-4) (7). The interactions between these molecules determines the fate of the cell (8). Notch receptor-ligand interactions lead to proteolytic cleavage of Notch by $\gamma$-secretase and other proteases, which causes the release of the Notch intracellular domain (NICD) from the plasma membrane and initiates its subsequent translocation into the nucleus. In the nucleus, the NICD binds to one of three cofactors, CBF-1/Suppressor of Hairless/Lag-1 (CSL), mastermind-like (MAML)-1 and p300/CBP, to generate a complex that acts as a transcriptional coactivator $(3,9)$. This complex then activates the transcription of target genes, such as Hes-1 and Hey-1 (10,11). Thus, chemotherapeutic agents that more specifically block the Notch signaling pathway to inhibit the proteolytic cleavage activity of $\gamma$-secretase would be an essential development in cancer therapy.

Niclosamide, an anthelminthic drug, has been used in the treatment of tapeworm infections for approximately five decades (12). Several studies have independently reported that niclosamide is active against cancer cells, including leukemia (13), colon cancer (14), glioblastoma (15) and breast 
cancer (16), although its precise mechanism of antitumor action remains only partially understood. Due to this lack of understanding, we performed this study in order to determine whether niclosamide triggers the inactivation of the Notch pathway, which in turn may contribute to the increased expression of members of the microRNA (miRNA or miR)-200 family that are suppressed in colon cancer.

Mounting evidence indicates that cross-talk occurs between miRNAs and the Notch signaling pathway during carcinogenesis (17). miRNAs are a class of small, evolutionary conserved, non-coding RNAs that bind to the $3^{\prime}$ untranslated region of messenger RNAs (mRNAs) in order to regulate gene expression (18-20). miRNAs are involved in various regulatory cellular functions including cell proliferation, differentiation and apoptosis (21). They regulate the Notch signaling pathway and Notch-related genes and affect various types of cancer (20). Moreover, some miRNAs have been reported to be oncomirs, which act either as oncogenes or as oncosuppressors (22). This indicates that further understanding of the Notch signaling-miRNA circuit may be critical for the discovery of novel chemotherapeutic agents for the treatment of colon cancer.

Five members of the miR-200 family, miR-200a, miR-200b, miR-200c, miR-141 and miR-429, which are located on chromosomes 1 and 12 in humans, have been identified $(20,23)$. miRNA-200 family members play a vital role in suppressing cancer metastasis by inhibiting the epithelial-mesenchymal transition (EMT). Decreased levels of miR-200c and miR-141 are associated with the increased expression of the transcription factor ZEB-1 (20), which in turn activates the Notch signaling pathway by targeting Notch ligand Jagged-1 and mastermind-like co-activators MAML-2 and -3 (24). Through suppression of the miRNA-200 family, ZEB-1 upregulates the Notch signaling pathway in tumor cells (24). ZEB-1 is an EMT activator, and its expression promotes tumor metastasis by inducing EMT activation (25). It has previously been shown that overexpression of the miRNA-200 family may inhibit EMT through increased E-cadherin expression targeting ZEB1 and ZEB2 (23). Low levels of miR-141 and miR-200c affect stem cell properties and drug resistance in pancreatic adenocarcinoma and basal breast cancer types (24). Furthermore, it has been shown that re-expression of miR-141 and miR-429 hinders Jagged-1 in metastatic prostate cancer cells, which suggests a novel means of controlling the fate of the Notch pathway (26). A study by Wang et al revealed that transfection of miR-200b in Rink-1 cells (pancreatic cell line) inactivated the Notch signaling pathway directly by decreasing the levels of Jagged-1/2 and those of their target genes Hes-1, Hey-2 and Bcl-2, which led to the inhibition of cell growth (27). Taken together, these findings show the connection among Notch signaling, miRNA-200 and ZEB in cancer. Nevertheless, a more in-depth investigation is warranted in order to understand how the miR-200 family directly regulates the Notch signaling pathway.

Collectively, these findings suggest that pharmacologic inactivation of Notch signaling with niclosamide may have potential therapeutic applications in the treatment of colon cancer. Herein, we report for the first time, to the best of our knowledge, a novel mechanism by which niclosamide inhibits colon cancer progression through upregulating the tumor suppressor miRNA-200 family and suppressing the Notch pathway. This strategy may be of therapeutic value in colon cancer and provide the basis for the development of specific inhibitors.

\section{Materials and methods}

Cell lines and cell culture. Human colon cancer cell lines LoVo and SW620 were purchased from the Cell Bank of the Shanghai Institutes for Biological Sciences, Chinese Academy of Sciences (Shanghai, China). All LoVo, SW620 and HCT116 (China Infrastructure of Cell Line Resources, Beijing, China) cells were grown in RPMI-1640 medium supplemented with $10 \%$ heat-inactivated fetal bovine serum (FBS) and $1 \%$ penicillin and streptomycin. The cell lines were maintained in a humidified incubator at $37^{\circ} \mathrm{C}$ with an atmosphere of $5 \% \mathrm{CO}_{2}$.

Reagents and antibodies. Niclosamide was obtained from Sigma-Aldrich(St.Louis,MO,USA).3-(4,5-Dimethylthiazol-2yl)-2,5-diphenyltetrazolium bromide (MTT; also known as thiazolyl blue and methylthiazolyldiphenyl tetrazolium bromide) was purchased from Sigma-Aldrich. The Annexin V-FITC Apoptosis Detection kit was purchased from Vazyme Biotech (Nanjing, China). Dimethyl sulfoxide (DMSO) was obtained from BioSharp (Hefei, China). The primary antibodies, rabbit polyclonal anti-Notch1 (ab27526), rabbit polyclonal anti-Notch2 (ab8926), rabbit polyclonal to anti-Notch3 (ab23426) and rabbit polyclonal anti-Heyl (ab22614) were purchased from Abcam (Cambridge, UK). Mouse anti- $\beta$-actin monoclonal antibody (TA-09) was purchased from ZSGB-BIO (Beijing, China). The secondary antibodies, peroxidase-conjugated AffiniPure goat anti-rabbit IgG (ZB-2301) and peroxidase-conjugated AffiniPure goat anti-mouse IgG (ZB-2305), were purchased from ZSGB-BIO.

Cell proliferation assay. For the cell proliferation assay, HCT116, LoVo and SW620 cells $\left(2-3 \times 10^{3}\right)$ were seeded in 96-well plates (one plate for each day) and incubated for $24 \mathrm{~h}$ to allow the cells to attach to the bottom of the wells. The cells were then treated with various concentrations of niclosamide or the corresponding volume of DMSO for 24, 48 and $72 \mathrm{~h}$. To determine cell proliferation, MTT was added at a final concentration of $0.5 \mathrm{mg} / \mathrm{ml}$ and incubated for $3 \mathrm{~h}$ at $37^{\circ} \mathrm{C}$ and $5 \% \mathrm{CO}_{2}$ in a humidified incubator. Crystallized MTT was resolved by 1:1 DMSO and isopropanol, and the absorption of each well was measured at $570 \mathrm{~nm}$ using a microplate spectrophotometer (xMark: BioRad, Berkeley, CA, USA). In addition, the morphology of the cells was observed under an inverted phase contrast microscope, and images were captured with the microscope (1X71; Olympus, Tokyo, Japan).

Wound-healing assay. For the wound-healing assay, HCT116, LoVo and SW620 cells were grown to confluence in 6-well plates. A perpendicular scratch wound was generated with a $200 \mu \mathrm{l}$ pipette tip, and the wells were washed twice with phosphate-buffered saline (PBS) to remove the detached cells; the cells were then incubated with $1 \mu \mathrm{M}$ niclosamide or DMSO for $48 \mathrm{~h}$. Images of each well were captured at 0 and $48 \mathrm{~h}$. Wound healing was quantified by measuring the distance between the wound edges using Image-Pro Plus 6.0 software. 
Transwell (Boyden chamber) migration assay. A Boyden chamber ( $8 \mathrm{~mm}$ pore size; Corning, Inc., Acton, MA, USA) was used to perform the assay. A total of $2.5 \times 10^{5}$ HCT116, LoVo or SW620 cells in $200 \mu 1$ serum free medium were added to the top chamber and treated with different concentrations of niclosamide. Then, $600 \mu 1$ medium with $20 \%$ FBS was added to the bottom chamber to act as the chemoattractant. The cells were allowed to migrate for $24 \mathrm{~h}$. Non-migrated cells in the top chamber were removed. The migrated cells were fixed in $4 \%$ paraformaldehyde and stained with $0.5 \%$ crystal violet (Biosharp, Hefei, China). The migrated cells were counted and images were captured under an inverted microscope.

Apoptotic assay. To examine the apoptosis inducing effect of niclosamide, an Annexin V-FITC apoptosis detection kit (Vazyme Biotech) was used. The cells, HCT116 and SW620 ( $4 \times 10^{5}$ cells/well) were seeded in a 6-well plate overnight and treated with niclosamide at the indicated concentrations. After the 48-h treatments, the cells were harvested and washed with cold PBS twice. Apoptosis levels were studied using the apoptosis detection kit according to the manufacturer's instructions by flow cytometry. The data were then analyzed using FlowJo software.

Western blot analysis. For total protein extraction, HCT116, LoVo and SW620 cells $\left(4 \times 10^{5}\right)$ were seeded overnight in 6 -well plates and treated with niclosamide. After $48 \mathrm{~h}$, the cells were lysed with a total protein extraction kit (KeyGen, Nanjing, China). To assess the total protein concentration, a BCA protein assay was performed using a BCA protein assay kit (Beyotime Biotechnology, Shanghai, China) according to the manufacturer's instructions. The lysates of equal protein concentrations were then separated by sodium dodecyl sulfate-polyacrylamide gel electrophoresis (SDS-PAGE) and transferred to nitrocellulose membranes using a wet transfer system. The membranes were incubated in blocking solution containing 5\% nonfat dry milk in Tris-buffered saline containing $0.1 \% \mathrm{v} / \mathrm{v}$ Tween-20 (TBST) for $1 \mathrm{~h}$ at room temperature. The membranes were incubated overnight at $4^{\circ} \mathrm{C}$ with rabbit anti-human Notch1 polyclonal antibodies (dilution, 1:500), rabbit anti-human Notch2 polyclonal antibodies (dilution, 1:1,000), rabbit anti-human Notch3 polyclonal antibodies (dilution, 1:500), rabbit anti-human Heyl polyclonal antibodies (dilution, 1:200) and mouse anti- $\beta$-actin polyclonal antibodies (dilution, 1:500), followed by incubation for $1 \mathrm{~h}$ at room temperature with HRP-conjugated anti-rabbit antibody (dilution, 1:5,000) or anti-mouse IgG antibody (dilution, 1:2,000). The antibody protein complexes were visualized with an electrochemical luminescence reagent (ECL system; Pierce; Thermo Fisher Scientific, Rockford, IL, USA) according to the manufacturer's instructions, and subsequent exposure to ImageQuant LAS 500 (GE Healthcare Life Sciences, Shanghai, China) for $30 \mathrm{sec}$ to $10 \mathrm{~min}$.

miRNA expression analysis. To determine the effect of niclosamide on the expression of the miR-200 family (miR-200a, miR-200b, miR-200c, miR-141 and miR-429) in colon cancer cells, the cells were seeded overnight in 6-well plates and treated with niclosamide. After $48 \mathrm{~h}$, total RNA from the cultured cells was extracted using TRIzol reagent
Table I. List of miRNAs and mature miRNA sequences used in this study.

\begin{tabular}{ll}
\hline miRNA & \multicolumn{1}{c}{ Mature miRNA sequence } \\
\hline hsa-miR-200a-3p-F & GCGCTAACACTGTCTGGTAACGATGT \\
hsa-miR-200b-3p-F & GCCGTAATACTGCCTGGTAATGATGA \\
hsa-miR-200c-3p-F & CGTAATACTGCCGGGTAATGATGGA \\
hsa-miR-141-3p-F & GCGGTAACACTGTCTGGTAAAGATGG \\
hsa-miR-429-3p-F & GCGGTAATACTGTCTGGTAAAACCGT \\
U6-F & CTCGCTTCGGCACA \\
U6-R & AACGCTTCACGAATTTGCGT \\
\hline
\end{tabular}

F, forward; R, reverse.

(Takara Biotech, Beijing, China) according to the manufacturer's instructions. To detect mature miRNA expression, reverse transcription-quantitative polymerase chain reaction (RT-qPCR) was performed. The extracted RNA was reverse transcribed, and cDNA was synthesized using an miRcute miRNA First-Strand cDNA synthesis kit (Tiangen Biotech, Beijing, China) according to the manufacturer's instructions. qPCR was then conducted with an miRcute miRNA qPCR detection kit (SYBR-Green I) (Tiangen Biotech). U6 was used as an endogenous control. The following PCR cycling conditions were used: $95^{\circ} \mathrm{C}$ for $30 \mathrm{sec}$ followed by 40 cycles of $95^{\circ} \mathrm{C}$ for $5 \mathrm{sec}$ and $60^{\circ} \mathrm{C}$ for $30 \mathrm{sec}$. In addition, melting curves were used to estimate non-specific amplification. The relative expression level was calculated using the $\Delta \Delta \mathrm{Ct}$ method. The primer sequences used in this study are presented in Table I.

Statistical analysis. All statistical analyses were performed using GraphPad Prism 5 software. A one-way analysis of variance (ANOVA) was used for comparisons among the groups, and a two-way ANOVA was used to compare two independent variables among the groups, followed by Tukey's test to compare all pairs of columns. The data are shown as the means \pm SE; $\mathrm{P}<0.05$ was considered to indicate a significant difference.

\section{Results}

Niclosamide affects the growth and morphology of colon cancer cells. To determine whether niclosamide exerts direct effects on colon cancer cells, we performed MTT assays to examine the inhibitory effect of niclosamide on the proliferation of different colon cancer cell lines. As shown in Fig. 1A, progressive inhibition of the growth of the HCT116, LoVo and SW620 cell lines occurred following treatment with niclosamide at concentrations of 1,2 and $4 \mu \mathrm{M}$ for different time periods (24, 48 and $72 \mathrm{~h}$ ). As shown in Fig. 1A, there was no significant difference in the viability of cells treated with $0.1 \%$ DMSO and the control cells grown with medium only; therefore, we used both as the controls for our subsequent experiments. Niclosamide significantly suppressed the proliferation of colon cancer cells after $24 \mathrm{~h}$ at a dose of $4 \mu \mathrm{M}$, which 

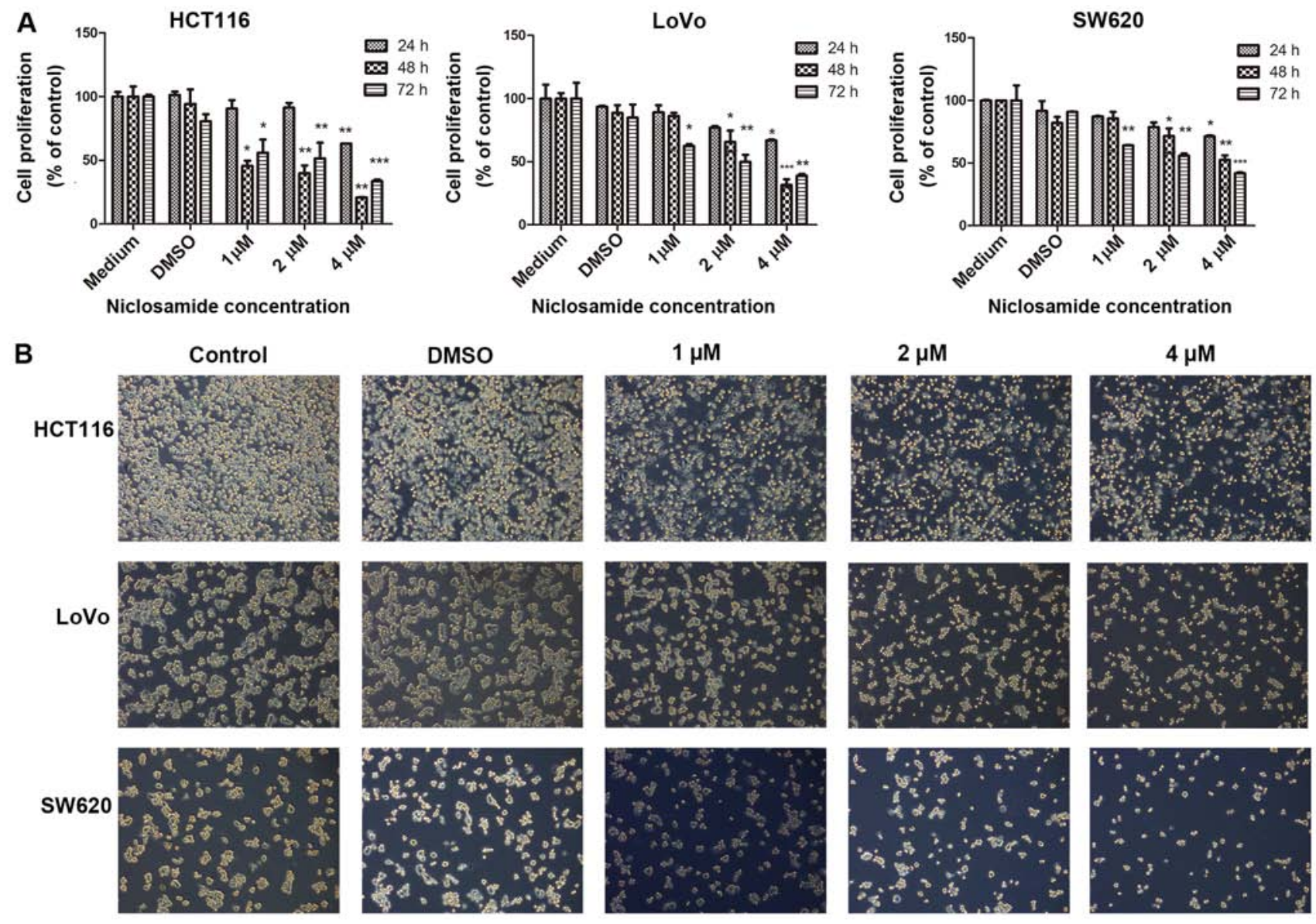

Figure 1. Effects of niclosamide on the proliferation and morphology of colon cancer cells. (A) Human colon cancer cell lines were treated with niclosamide at the indicated doses for 1-3 days and the proliferation rate was measured using the MTT assay. Niclosamide treatment resulted in dose- and time-dependent inhibition of cell growth. (B) Colon cancer cells were seeded in 6-well plates overnight and treated with niclosamide for 24 h and images were captured using an inverted microscope (magnification, x100). Significant changes in cell morphology were observed in the niclosamide-treated groups. The results are expressed as the means $\pm \mathrm{SE}\left({ }^{*} \mathrm{P}<0.05 ;{ }^{* *} \mathrm{P}<0.01\right.$ and ${ }^{* * *} \mathrm{P}<0.001$ vs. DMSO).

was followed by further significant suppression over the next 48 and $72 \mathrm{~h}$ in a dose- and time-dependent manner. The analysis of cell morphology revealed that niclosamide treatment resulted in a dose-dependent change in cellular morphology, with a stretched morphology at lower niclosamide concentrations and a rounded morphology at higher niclosamide concentrations (Fig. 1B).

Niclosamide induces the apoptosis of colon cancer cells. It has been reported that niclosamide induces the apoptosis of breast cancer cells (16). To determine whether niclosamide induced the apoptosis of colon cancer cells, we performed an apoptosis assay using the Annexin V-FITC apoptosis detection kit. HCT116 and SW620 cells were treated with 1, 2 and $4 \mu \mathrm{M}$ niclosamide, with medium and DMSO serving as controls, for $48 \mathrm{~h}$. Following treatment, the apoptosis-inducing effect of niclosamide was clearly observed in both cell lines (Fig. 2). The induction of apoptosis was dose-dependent. Specifically, niclosamide at concentrations of 1,2 and $4 \mu \mathrm{M}$ induced apoptosis rates of $16 \pm 2.763,26.13 \pm 5.095$ and $32.05 \pm 9.015 \%$, respectively, in the HCT116 cells. Similarly, niclosamide at concentrations of 1,2 and $4 \mu \mathrm{M}$ triggered apoptosis at rates of $8.10 \pm 1.945,8.87 \pm 0.9300$ and $11.40 \pm 0.2000 \%$, respectively, in the SW620 cells. These results clearly indicate that niclosamide induces the apoptosis of colon cancer cells.
Niclosamide decreases the migration of colon cancer cells. Tumor cell migration remains one of the key steps in successful cancer metastasis. Therefore, to determine whether niclosamide inhibits colon cancer cell migration, we conducted wound-healing assays on HCT116, SW620 and LoVo cells. As shown in Fig. 3A, niclosamide treatment at a concentration of $1 \mu \mathrm{M}$, significantly decreased the wound healing capacity of HCT116, SW620 and LoVo cells compared with the control cells after $48 \mathrm{~h}$. In the HCT116 cells, niclosamide treatment decreased wound closure by $92.337 \pm 1.356 \%$ compared with the control. Niclosamide treatment inhibited wound closure by $52.85 \pm 2.129 \%$ in SW620 cells. Similarly, niclosamide treatment in LoVo cells decreased their wound healing ability by $78.19 \pm 0.4531 \%$ (Fig. 3B). To confirm these results, we also performed a Transwell migration assay and found that niclosamide inhibited the migration of the HCT116, SW620 and LoVo cells after 24 h (Fig. 3C). At a $1 \mu \mathrm{M}$ concentration, niclosamide significantly decreased migration by $49.26 \pm 4.412 \%$ in the HCT116 cells compared with the control (Fig. 3D). In addition, niclosamide decreased migration by $54.49 \pm 6.696 \%$ in the SW620 cells (Fig. 3E). Furthermore, in the LoVo cells, migration was inhibited by $54.49 \pm 6.696 \%$ with $1 \mu \mathrm{M}$ niclosamide (Fig. $3 \mathrm{~F}$ ). These results show that niclosamide suppresses the migration of colon cancer cells. 


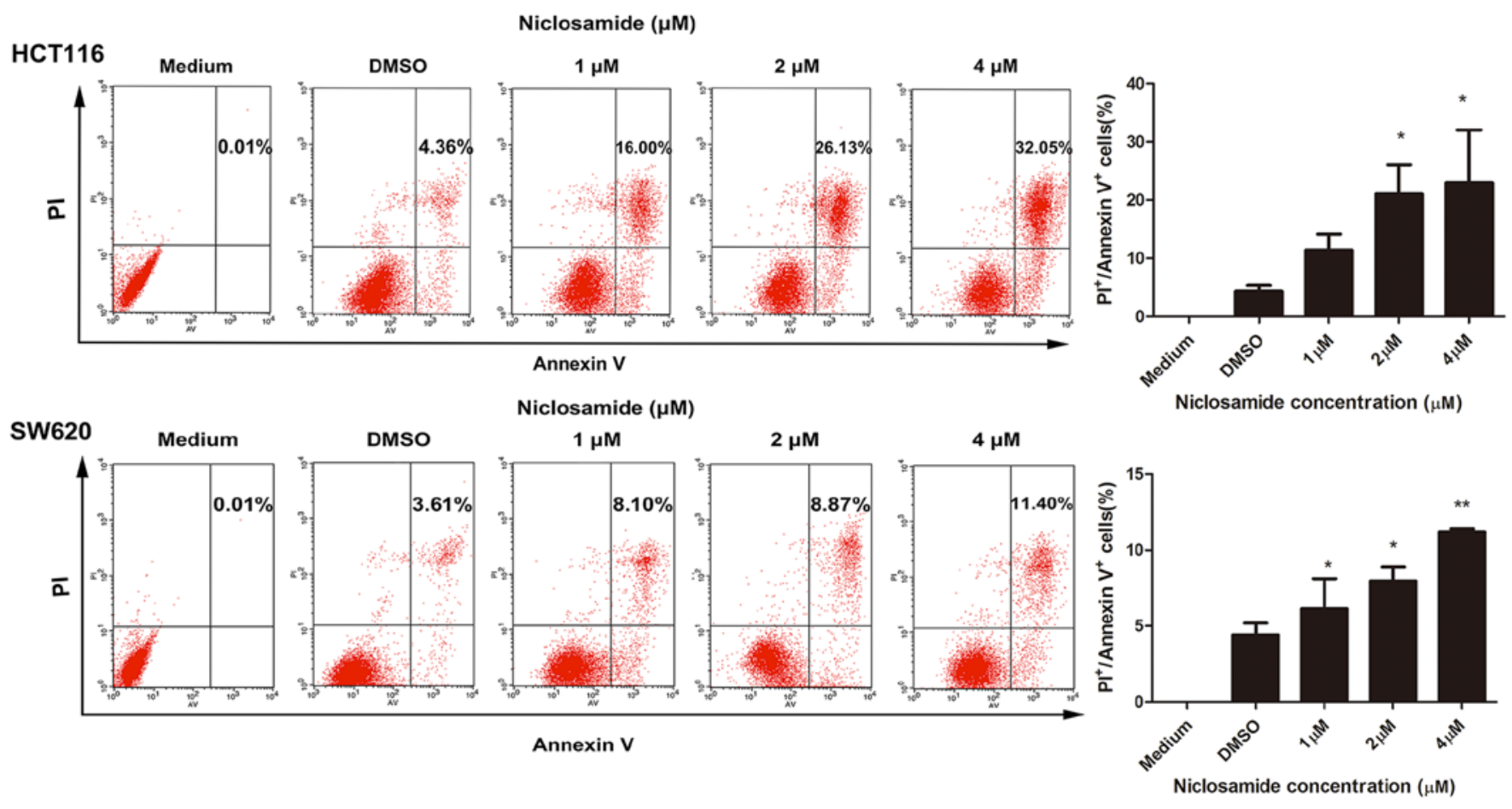

Figure 2. Niclosamide induces the apoptosis of colon cancer cells. HCT116 and SW620 cells were treated with niclosamide at the indicated doses for 48 h, and the level of apoptosis was assessed using the Annexin V/PI dual-labeling technique, as determined by flow cytometry (FCM). Data shown are representative of two independent experiments. Data are expressed as means $\pm \mathrm{SE}$ from two independent experiments $\left({ }^{*} \mathrm{P}<0.05\right.$ and ${ }^{* *} \mathrm{P}<0.01$ vs. DMSO).

Niclosamide downregulates the Notch signaling pathway. Previous studies have indicated that the Notch pathway regulates cell proliferation and migration and induces apoptosis during cancer development $(28,29)$. In this study, we examined whether niclosamide affected the Notch pathway to inhibit the proliferation, decrease the migration and induce the apoptosis of colon cancer cells. The expression of Notch1, Notch2, Notch3 and the downstream target gene Hey-1 in HCT116, SW620 and LoVo cells was assessed using western blot analysis following the 48-h treatment of colon cancer cells with different niclosamide concentrations (1, 2 and $4 \mu \mathrm{M})$. Western blot analysis demonstrated that niclosamide treatment resulted in reduced levels of the Notch receptors and Heyl in the colon cancer cells (Fig. 4).

Effect of niclosamide on the expression of miRNAs in colon cancer cells. Previous studies have shown that the miR-200 family plays a role in the carcinogenesis of colon cancer (30) and that cross-talk occurs between miRNAs and Notch signaling pathways during the development and progression of tumors (17). Therefore, we investigated whether niclosamide treatment regulated the expression of the miR-200 family (miR-200a, miR-200b, miR-200c, miR-141 and miR-429) in colon cancer cells using RT-qPCR. Following a 48-h treatment of the colon cancer cells with niclosamide at a concentration of $2 \mu \mathrm{M}$, we found that niclosamide treatment increased the relative expression of miR-200a and miR-141 in the HCT116, SW620 and LoVo colon cancer cells (Fig. 5). Niclosamide increased the relative expression of miR-200b and miR-200c in the LoVo and SW620 cells but not in the HCT116 cells. Notably, niclosamide treatment increased the relative expression of miR-429 only in the LoVo cells but not in the HCT116 and SW620 cells. These data indicate that niclosamide differ- entially regulates the expression of these tumor-associated miRNAs in colon cancer cells.

\section{Discussion}

$\mathrm{CRC}$ has become a major problem and is one of the most frequent causes of cancer-related death worldwide (1). It remains incurable, which indicates the need for novel therapeutic strategies. For nearly five decades, niclosamide has been used for the treatment of intestinal parasite infections in humans (31). Notably, a number of studies have shown that it also has potential anticancer activity in both in vitro and in vivo experiments through the inactivation of multiple signaling pathways $(13,38)$; however, the underlying molecular mechanisms remain poorly understood. In the present study, we aimed to explore the potential anticancer activity of niclosamide in three colon cancer cell lines (LoVo, SW620 and HCT116) and to elucidate the underlying molecular mechanism responsible for the suppressive effects of niclosamide in colon cancer. Under normal conditions, the activated Notch signaling pathway plays a vital role in controlling the apoptosis, differentiation and proliferation of cells (32). Accumulating evidence indicates that inappropriate activation of the Notch signaling plays a critical role in the pathogenesis of cancer (33). Recent studies have revealed that Notch receptors and their ligands are aberrantly activated in many types of human cancers, including colon cancer $(34,35)$. Thus, considerable effort has been made to block this pathway with an increasing range of pharmacologic agents, primarily through the inhibition of Notch cleavage (36). Therefore, the downregulation of the Notch signaling pathway may be a novel approach for the treatment of colon cancer. 
A

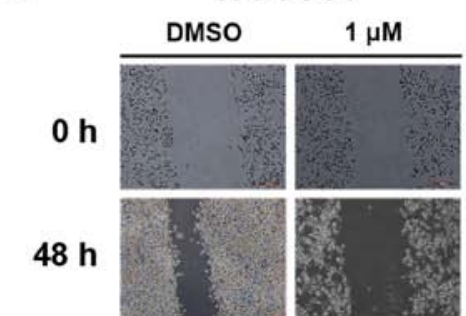

C

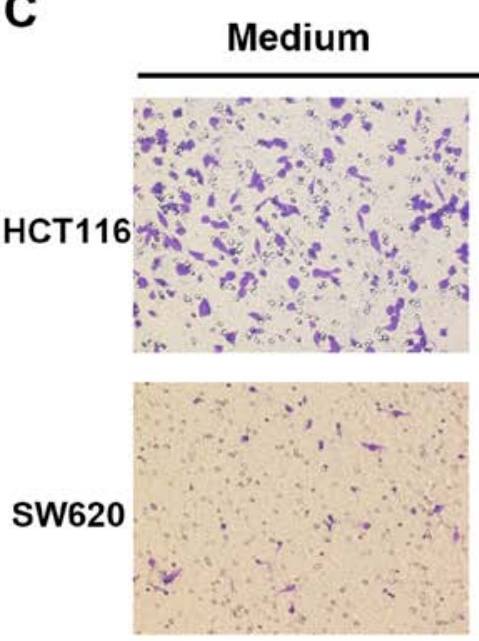

LoVo

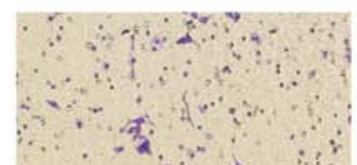

ind
SW620

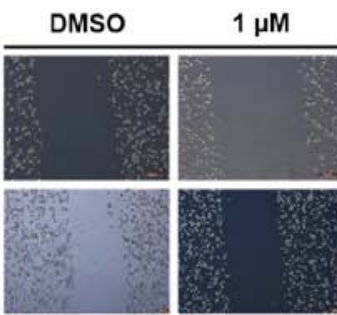

DMSO
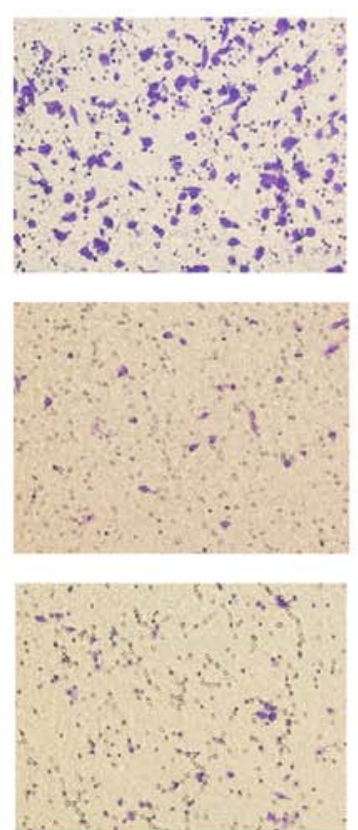

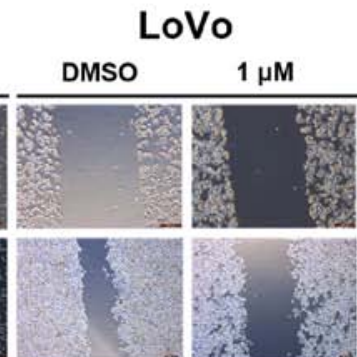

$1 \mu \mathrm{M}$
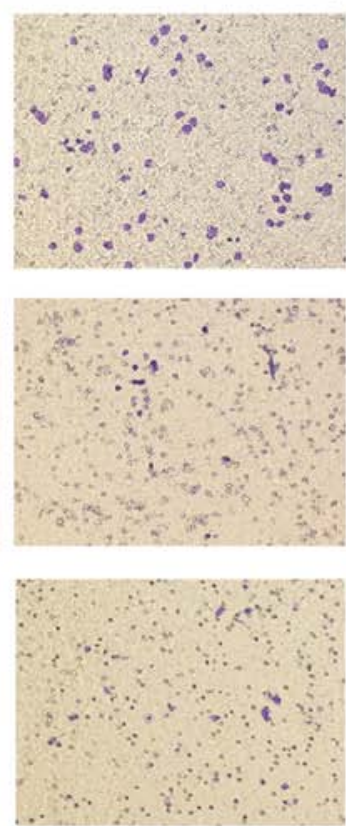

B
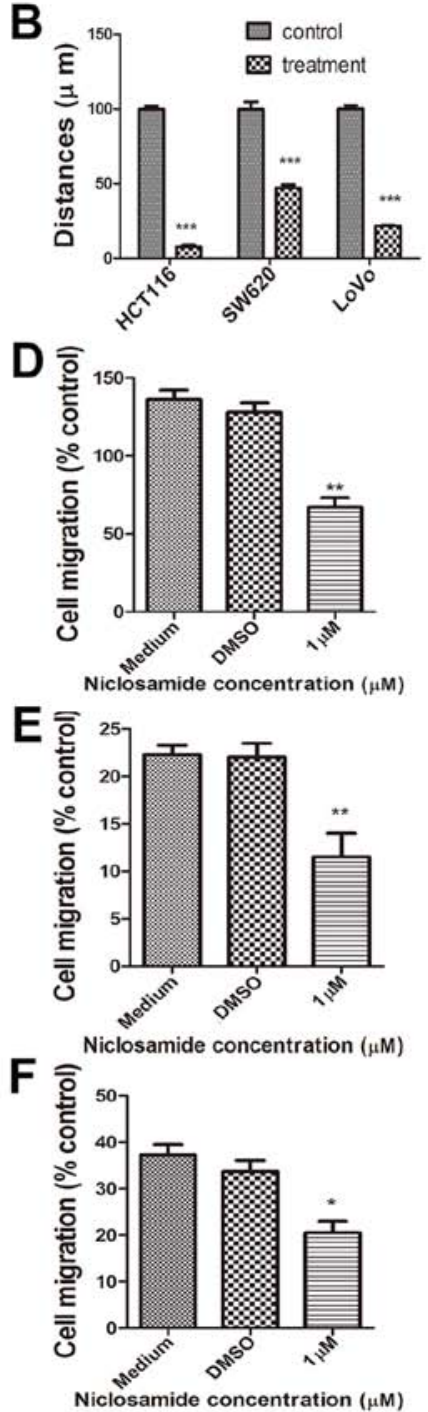

Figure 3. Niclosamide decreases the migration of HCT116, SW620 and LoVo colon cancer cells. (A) Niclosamide reduced cell migration as determined by wound-healing analysis. (B) Statistical results of wound-healing assay. (C) Niclosamide inhibits cell migration, as determined by a Transwell assay and crystal violet staining. HCT116, SW620 and LoVo cells were seeded in a Boyden chamber and treated with niclosamide for $48 \mathrm{~h}$ and $20 \%$ FBS acted as the chemoattractant for the migrating cancer cells. (D-F) Statistical results of Transwell migration assay for HCT116, SW620 and LoVo cells, respectively. Values are the means \pm SE of three determinations $\left({ }^{*} \mathrm{P}<0.05,{ }^{* *} \mathrm{P}<0.01\right.$ and ${ }^{* * *} \mathrm{P}<0.001$ vs. DMSO).
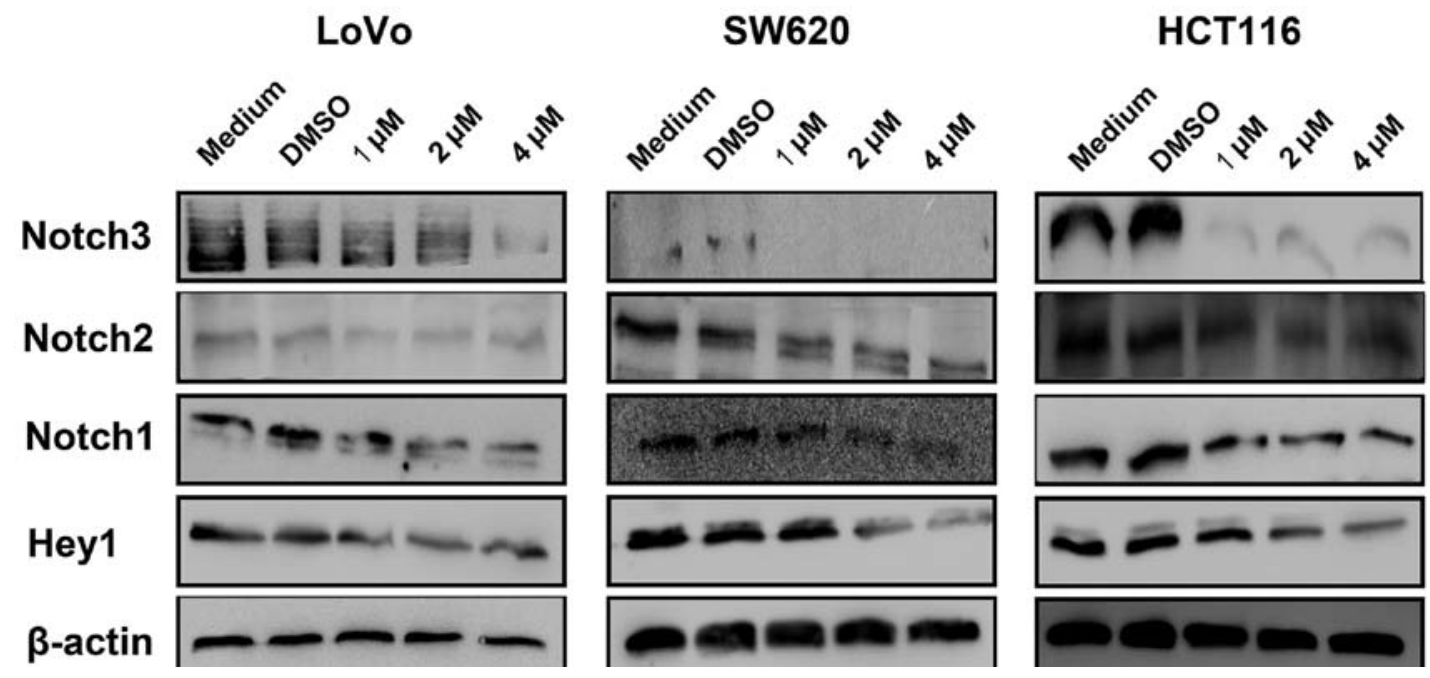

Figure 4. Effects of niclosamide on Notch signaling pathway in colon cancer cells. LoVo, SW620 and HCT116 cells were seeded in 6-well plates and treated with niclosamide at the indicated concentrations for $48 \mathrm{~h}$. Whole cell lysates were prepared and blotted with specific antibodies (anti-Notch1, anti-Notch2, anti-Notch3 and anti-Heyl). All the samples were also probed with $\beta$-actin antibody to verify equal loading. 

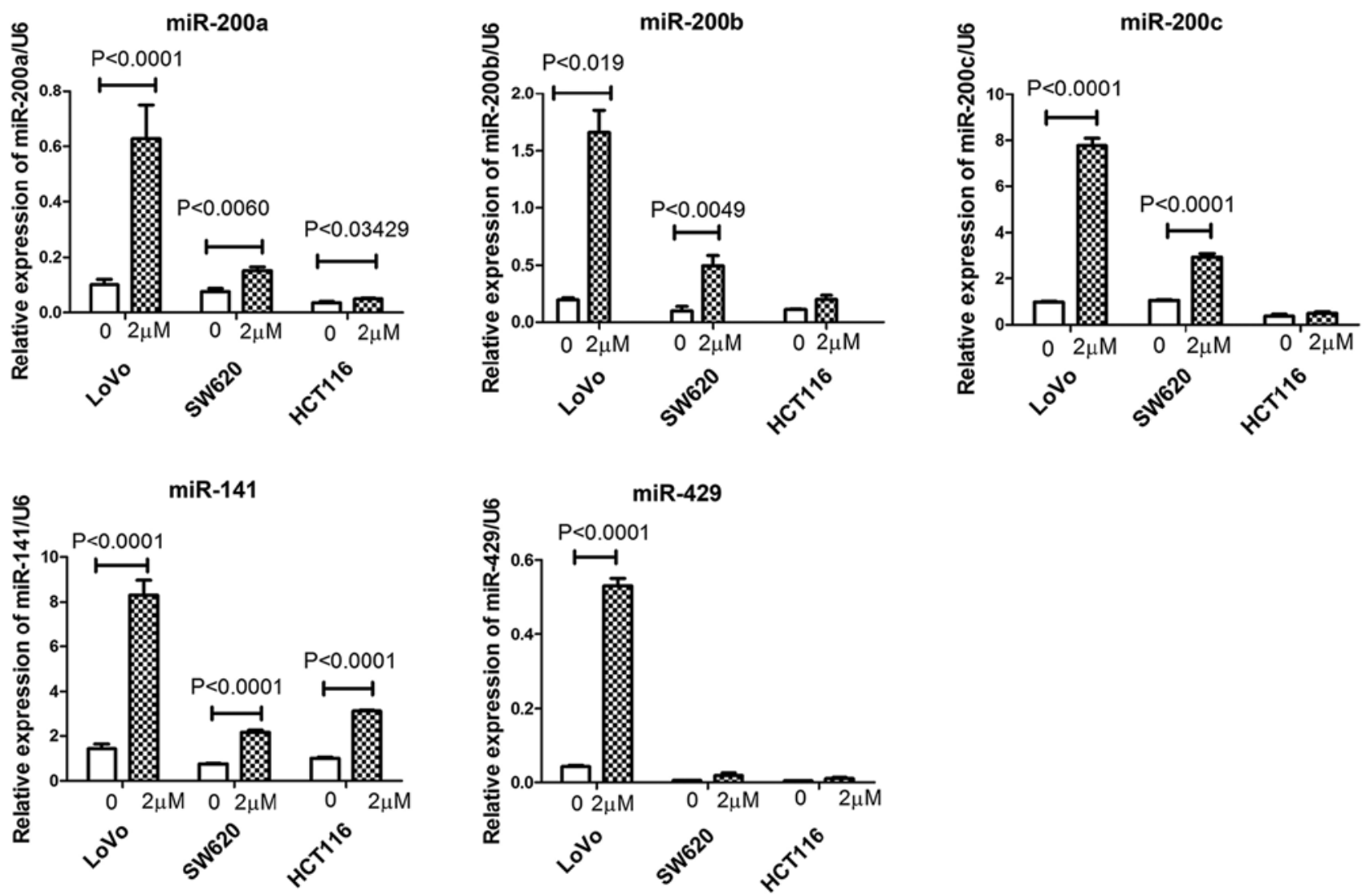

Figure 5. Effects of niclosamide on the tumor suppressor miR-200 family. Niclosamide upregulated the expression of miR-200a, miR-200b, miR-200c, miR-141 and miR-429. RT-qPCR of the miRNAs was performed as described in the Materials and methods section. RNU6 was used to normalize the mRNA level. Data are expressed as the means $\pm \mathrm{SE}$ from three independent experiments.

Multiple studies by different groups have shown that niclosamide inhibits several molecules and signaling pathways, including the nuclear factor- $\mathrm{\kappa B}(\mathrm{NF}-\kappa \mathrm{B})$ signaling pathway (13), the mTOR-signaling pathway (37), the Wnt/ $\beta$-catenin signaling pathway (38), the signal transducers and activators of the transcription 3 (Stat3) signaling pathway (16), and the Notch signaling pathway (39). However, the effects of niclosamide on colon cancer cells and the mechanisms responsible for these effects are not completely understood. In this study, we observed that colon cancer cells exposed to niclosamide treatment experienced growth inhibition and apoptosis induction, as demonstrated by the MTT assay and Annexin V-FITC apoptosis detection assay, respectively, which suggests that this treatment has therapeutic implications for the management of colon cancer. To explore the mechanism of increased apoptosis induced by niclosamide, we investigated the activity of the Notch pathway, which plays a key role in inhibiting the apoptotic response. We demonstrated for the first time, to the best of our knowledge, that niclosamide downregulates the expression of the Notch receptors (Notch1, Notch2 and Notch3) as well as the downstream target gene Heyl, which is likely to inhibit the development of colon cancer. Thus, we hypothesized that the possible novel mechanism responsible for the triggering of apoptosis by niclosamide may be due to the inhibition of the Notch gene expression. Notch signaling genes are functionally active and highly expressed, and suppress apoptosis and promote cell growth in colon cancer $(5,40)$. Moreover, the results of the Transwell assay showed that niclosamide, at a low concentration of $1 \mu \mathrm{M}$, inhibited the migration of colon cancer cells in vitro, which suggests that it may be a possible candidate for treating colon cancer metastasis. As the activation of Notch signaling may affect the upregulation of various other signaling pathways, such as the NF- $\mathrm{BB}, \mathrm{PI} 3 \mathrm{~K} / \mathrm{AKT}$, c-MYC and EGFR pathways (41), an effective and in-depth mechanistic study of the effects of niclosamide on these pathways through Notch signaling may be crucial for the design of novel therapeutic approaches for the treatment of colon cancer. In this regard, our findings may serve as a base for further study of the anti-colon cancer effects of niclosamide due to the inactivation of Notch signaling affecting multiple target genes in these pathways.

miRNAs are key players that function as endogenous post-transcriptional gene controllers to mediate protein synthesis or mRNA stability $(18,19)$. It has been demonstrated that a large number of miRNAs are associated with tumor growth and progression by regulating the expression and transcription of many tumor-associated genes $(22,42,43)$. Several studies have suggested that miR-200 serves as a potential tumor suppressor, primarily by repressing the acquisition of the EMT phenotype during tumor development and progression $(44,45)$. The decreased expression of miR-200 has been observed in many tumors, such as pancreatic, breast and prostate cancer, which is associated with tumor invasion and metastasis (46). For the first time, to the best of our knowledge, this study demonstrated that the miR-200 family was downregulated in colon cancer cells and that inactivation of the Notch pathway by niclosamide 
led to increased expression of the miR-200 family (miR-200a, miR-200b, miR-200c, miR-141 and miR-429). This suggests that the Notch pathway is involved in the regulation of the miR-200 family, and that niclosamide may induce the re-expression of these miRNAs, which would be highly valuable in attenuating the aggressiveness of tumor cells.

In conclusion, these experiments provide mechanistic evidence that the Notch pathway is inhibited in colon cancer cells in response to niclosamide treatment. The downregulation of Notch signaling by niclosamide is accompanied by the upregulation of tumor suppressor miRNAs (miR-200a, miR200b, miR-200c, miR-141 and miR-429). Therefore, we propose that the inhibition of Notch signaling is a novel strategy that may be used to impede the induction of cancer survival mechanisms in colon cancer. However, further in-depth, preclinical experiments using appropriate animal models are warranted.

\section{Acknowledgements}

The present study was supported by grants from the Chinese National Science Foundation Projects (nos. 81372669, 31270867 and 31470800), the Chinese State Key Program in Basic Research (no. 2012CB822103), the Science and Technology Planning Project of Liaoning province, China (no. 2012225020), and the Project of Chinese Ministry of Health (no. W2012RQ23).

\section{References}

1. Brenner H, Kloor M and Pox CP: Colorectal cancer. Lancet 383: 1490-1502, 2014.

2. Miyamoto S, Nakanishi M and Rosenberg DW: Suppression of colon carcinogenesis by targeting Notch signaling. Carcinogenesis 34: 2415-2423, 2013.

3. Katoh M and Katoh M: Notch signaling in gastrointestinal tract (Review). Int J Oncol 30: 247-251, 2007.

4. Prasetyanti PR, Zimberlin CD, Bots M, Vermeulen L, Melo FS and Medema JP: Regulation of stem cell self-renewal and differentiation by Wnt and Notch are conserved throughout the adenoma-carcinoma sequence in the colon. Mol Cancer 12: 126, 2013.

5. Reedijk M, Odorcic S, Zhang H, Chetty R, Tennert C, Dickson BC, Lockwood G, Gallinger S and Egan SE: Activation of Notch signaling in human colon adenocarcinoma. Int J Oncol 33: 1223-1229, 2008

6. Zhang Y, Li B, Ji ZZ and Zheng PS: Notch1 regulates the growth of human colon cancers. Cancer 116: 5207-5218, 2010.

7. Mumm JS and Kopan R: Notch signaling: from the outside in. Dev Biol 228: 151-165, 2000.

8. Li JL and Harris AL: Notch signaling from tumor cells: a new mechanism of angiogenesis. Cancer Cell 8: 1-3, 2005.

9. Iso T, Kedes L and Hamamori Y: HES and HERP families: multiple effectors of the Notch signaling pathway. J Cell Physiol 194: 237-255, 2003.

10. Bhanot U, Köhntop R, Hasel C and Möller P: Evidence of Notch pathway activation in the ectatic ducts of chronic pancreatitis. J Pathol 214: 312-319, 2008.

11. Ehebauer M, Hayward P and Arias AM: Notch, a universal arbiter of cell fate decisions. Science 314: 1414-1415, 2006.

12. Garin JP, Despeignes J and Billerau M: Present treatment of taeniasis with niclosamide. Lyon Med 212: 1581-1588, 1964 (In French).

13. Jin Y, Lu Z, Ding K, Li J, Du X, Chen C, Sun X, Wu Y, Zhou J and Pan J: Antineoplastic mechanisms of niclosamide in acute myelogenous leukemia stem cells: inactivation of the NF-kappaB pathway and generation of reactive oxygen species. Cancer Res 70: 2516-2527, 2010

14. Sack U, Walther W, Scudiero D, Selby M, Kobelt D, Lemm M, Fichtner I, Schlag PM, Shoemaker RH and Stein U: Novel effect of antihelminthic niclosamide on S100A4-mediated metastatic progression in colon cancer. J Natl Cancer Inst 103: 1018-1036, 2011.
15. Wieland A, Trageser D, Gogolok S, Reinartz R, Höfer H, Keller M, Leinhaas A, Schelle R, Normann S, Klaas L, et al: Anticancer effects of niclosamide in human glioblastoma. Clin Cancer Res 19: 4124-4136, 2013.

16. Ye T, Xiong Y, Yan Y, Xia Y, Song X, Liu L, Li D, Wang N, Zhang L, Zhu Y, et al: The anthelmintic drug niclosamide induces apoptosis, impairs metastasis and reduces immunosuppressive cells in breast cancer model. PLoS One 9: e85887, 2014.

17. Wang Z, Li Y, Kong D, Ahmad A, Banerjee S and Sarkar FH: Cross-talk between miRNA and Notch signaling pathways in tumor development and progression. Cancer Lett 292: 141-148, 2010.

18. Bartel DP: MicroRNAs: Genomics, biogenesis, mechanism, and function. Cell 116: 281-297, 2004.

19. Denli AM, Tops BB, Plasterk RH, Ketting RF and Hannon GJ: Processing of primary microRNAs by the Microprocessor complex. Nature 432: 231-235, 2004.

20. Prokopi M, Kousparou CA and Epenetos AA: The secret role of microRNAs in cancer stem cell development and potential therapy: a Notch-pathway approach. Front Oncol 4: 389, 2015.

21. Srinivasan S, Selvan ST, Archunan G, Gulyas B and Padmanabhan P: MicroRNAs -the next generation therapeutic targets in human diseases. Theranostics 3: 930-942, 2013.

22. Esquela-Kerscher A and Slack FJ: Oncomirs - microRNAs with a role in cancer. Nat Rev Cancer 6: 259-269, 2006.

23. Korpal $M$ and Kang Y: The emerging role of miR-200 family of microRNAs in epithelial-mesenchymal transition and cancer metastasis. RNA Biol 5: 115-119, 2008.

24. Brabletz S, Bajdak K, Meidhof S, Burk U, Niedermann G, Firat E, Wellner U, Dimmler A, Faller G, Schubert J and Brabletz T: The ZEB1/miR-200 feedback loop controls Notch signalling in cancer cells. EMBO J 30: 770-782, 2011.

25. Burk U, Schubert J, Wellner U, Schmalhofer O, Vincan E, Spaderna $S$ and Brabletz T: A reciprocal repression between ZEB1 and members of the miR-200 family promotes EMT and invasion in cancer cells. EMBO Rep 9: 582-589, 2008.

26. Vallejo DM, Caparros E and Dominguez M: Targeting Notch signalling by the conserved miR-8/200 microRNA family in development and cancer cells. EMBO J 30: 756-769, 2011.

27. Wang Z, Banerjee S, Ahmad A, Li Y, Azmi AS, Gunn JR, Kong D, Bao B, Ali S, Gao J, et al: Activated K-ras and INK4a/Arf deficiency cooperate during the development of pancreatic cancer by activation of Notch and NF- $\mathrm{kB}$ signaling pathways. PLoS One 6: e20537, 2011

28. Ferrari-Toninelli G, Bonini SA, Uberti D, Buizza L, Bettinsoli P, Poliani PL, Facchetti F and Memo M: Targeting Notch pathway induces growth inhibition and differentiation of neuroblastoma cells. Neuro Oncol 12: 1231-1243, 2010.

29. Rasul S, Balasubramanian R, Filipović A, Slade MJ, Yagüe E and Coombes RC: Inhibition of gamma-secretase induces $\mathrm{G} 2 / \mathrm{M}$ arrest and triggers apoptosis in breast cancer cells. Br J Cancer 100: 1879-1888, 2009.

30. Senfter D, Holzner S, Kalipciyan M, Staribacher A, Walzl A, Huttary N, Krieger S, Brenner S, Jäger W, Krupitza G, et al: Loss of miR-200 family in 5-fluorouracil resistant colon cancer drives lymphendothelial invasiveness in vitro. Hum Mol Genet 24: 3689-3698, 2015.

31. Li Y, Li PK, Roberts MJ, Arend RC, Samant RS and Buchsbaum DJ: Multi-targeted therapy of cancer by niclosamide: a new application for an old drug. Cancer Lett 349: 8-14, 2014.

32. Pastò A, Serafin V, Pilotto G, Lago C, Bellio C, Trusolino L, Bertotti A, Hoey T, Plateroti M, Esposito G, et al: NOTCH3 signaling regulates MUSASHI-1 expression in metastatic colorectal cancer cells. Cancer Res 74: 2106-2118, 2014.

33. Ranganathan P, Weaver KL and Capobianco AJ: Notch signalling in solid tumours: a little bit of everything but not all the time. Nat Rev Cancer 11: 338-351, 2011.

34. Dai Y, Wilson G, Huang B, Peng M, Teng G, Zhang D, Zhang R, Ebert MP, Chen J, Wong BC, et al: Silencing of Jagged1 inhibits cell growth and invasion in colorectal cancer. Cell Death Dis 5: e1170, 2014.

35. Ozawa T, Kazama S, Akiyoshi T, Murono K, Yoneyama S, Tanaka T, Tanaka J, Kiyomatsu T, Kawai K, Nozawa H, et al: Nuclear Notch3 expression is associated with tumor recurrence in patients with stage II and III colorectal cancer. Ann Surg Oncol 21: 2650-2658, 2014.

36. Shih IeM and Wang TL: Notch signaling, gamma-secretase inhibitors, and cancer therapy. Cancer Res 67: 1879-1882, 2007. 
37. Balgi AD, Fonseca BD, Donohue E, Tsang TC, Lajoie P, Proud CG, Nabi IR and Roberge M: Screen for chemical modulators of autophagy reveals novel therapeutic inhibitors of mTORC1 signaling. PLoS One 4: e7124, 2009.

38. Osada T, Chen M, Yang XY, Spasojevic I, Vandeusen JB, Hsu D, Clary BM, Clay TM, Chen W, Morse MA and Lyerly HK: Antihelminth compound niclosamide downregulates Wnt signaling and elicits antitumor responses in tumors with activating APC mutations. Cancer Res 71: 4172-4182, 2011.

39. Wang AM, Ku HH, Liang YC, Chen YC, Hwu YM and Yeh TS: The autonomous notch signal pathway is activated by baicalin and baicalein but is suppressed by niclosamide in K562 cells. J Cell Biochem 106: 682-692, 2009.

40. Fernández-Majada V, Aguilera C, Villanueva A, Vilardell F, Robert-Moreno A, Aytés A, Real FX, Capella G, Mayo MW, Espinosa L and Bigas A: Nuclear IKK activity leads to dysregulated notch-dependent gene expression in colorectal cancer. Proc Natl Acad Sci USA 104: 276-281, 2007.

41. Qiao L and Wong BC: Role of Notch signaling in colorectal cancer. Carcinogenesis 30: 1979-1986, 2009.
42. Jones KB, Salah Z, Del Mare S, Galasso M, Gaudio E, Nuovo GJ, Lovat F, LeBlanc K, Palatini J, Randall RL, et al: miRNA signatures associate with pathogenesis and progression of osteosarcoma. Cancer Res 72: 1865-1877, 2012.

43. Kobayashi E, Hornicek FJ and Duan Z: MicroRNA involvement in osteosarcoma. Sarcoma 2012: 359739, 2012.

44. Kong D, Li Y, Wang Z, Banerjee S, Ahmad A, Kim HR and Sarkar FH: miR-200 regulates PDGF-D-mediated epithelial-mesenchymal transition, adhesion, and invasion of prostate cancer cells. Stem Cells 27: 1712-1721, 2009.

45. Li Y, VandenBoom TG II, Kong D, Wang Z, Ali S, Philip PA and Sarkar FH: Up-regulation of miR-200 and let-7 by natural agents leads to the reversal of epithelial-to-mesenchymal transition in gemcitabine-resistant pancreatic cancer cells. Cancer Res 69: 6704-6712, 2009

46. Zhang B, Pan X, Cobb GP and Anderson TA: microRNAs as oncogenes and tumor suppressors. Dev Biol 302: 1-12, 2007. 